ISSN 1991- 8690

website :http:// jsci.utq.edu.iq

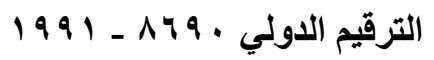

Email: utjsci@utq.edu.iq

\title{
Collision of Cluster of Ions with the Surfaces
}

\author{
F.H.Hanon ${ }^{(1)}$ \\ S.I.Easa ${ }^{(2)}$ \\ (1) Physics Department - Colledge of Science - Thi-Qar University \\ (2) Physics Department - Colledge of Education - Basrah University
}

\section{Abstract}

We study theoretically the collision between cluster of $\mathrm{Pt}$ and $\mathrm{Pb}$ ions with $\mathrm{Al}$ and graphite (HOPG) target in the ion incident range of energies within the range $(200 \mathrm{eV}-600 \mathrm{eV})$ and the cluster of $\mathrm{N}(1-10)$ ions. Using the rate equations, that describes the occupation numbers of the projectile and surface levels. Our results are in good agreement with experimental data.

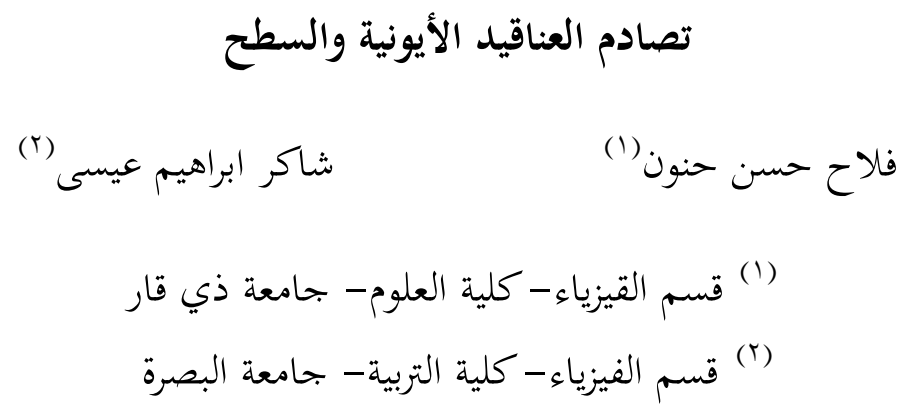

الملخصنا نظرياً التصادم بين عناقيد من ايونات Pb و Pb مع اهداف من الألمنيوم والكر افيت في مدى طاقة سقوط للأيون ضمن المدى (200eV - 600eV) السطح. نتائجنا هي في تو افق جيد مع القياسات العملية. 


\section{Introduction}

When an atom or molecule is close to a solid surface, its electronic state interacts with those of the solid, leading to the possibility of charge transfer between the atom (molecule) and the solid. This charge transfer process plays an important role in a variety of different situations. In particular, it often occurs as an intermediate step in reactions at surface (desorption, fragmentation of adsorbates, chemical reactions, etc.). The one-electron transfer between energetically degenerate electronic levels of the atom and the solid is called the resonant charge transfer (RCT) process [1-6], which is an effective one. The specific mechanisms those are relevant to ion-surface interactions made us to use the ion scattering as a probe of surface electronic properties.

Experimentally, if an ion sent against the surface it can neutralize by detaching electron from the surface. This process of the ion neutralization during scattering from surface has been the subject of much experimental [7][8] and theoretical [9-15] interests. Since, the neutralizing electron originates in the valance band of the solid; the process is closely related to the solid-state nature of the target. In this case, one can consider the effect of the surface density of state on the electron transfer process. And it turns out that the whole process can be considered as a probe for the surface density of state structure.

Charge exchange between a metal surface and an atom, or ion, has been observed in many ion (atom)surface scattering experiments [16-21]. For example, if the incoming particles are $\mathrm{H}\left(\right.$ or $\mathrm{He}^{+}$), then the scattered beam may contains $\mathrm{He}$ and $\mathrm{He}^{+}\left(\right.$or $\mathrm{H}^{-}, \mathrm{H}^{+}$and $\left.\mathrm{H}\right)$. The distributions of the scattered particles among the various charged states are strongly dependent on the velocity of the incoming particles and the nature of the surface. Therefore, experiments of this kind are important in many techniques of surface analysis, such as ion-beam scattering spectroscopy (ISS), neutral-beam scattering spectroscopy (NSS) and secondary ion mass spectroscopy (SIMS) [22-24].

When the cluster collides with a solid surface many different processes can be take place, depending on which of the relaxation channels become active. The redistribution, during and after the collision, of the initial kinetic energy of the cluster can result, for instance, in deformations (bond breaking), cluster fragmentation, creation of phonons and emission of atoms, ions, electrons and photons [25].

However, there are many studies (experimental and theoretical) focused on the cluster surface collision at low and moderate energies [25]. The importance of these studies comes from the needing to modify the surface by using cluster bombardment or surface deposition, light emission, particle emission, cluster impact chemistry and femtosecond tunneling of electrons. The incident bombardment energies need to keep surface without any damage, so these energies lie between low and moderate range in which one can expect the electronic quantum effect. The neutralization dynamics depends on many factors such that cluster type, cluster size, target, etc because these factors effect on the charge exchange between cluster and surface, where charged cluster can be neutralized or there charges can be fluctuate during the collision.

The present theoretical work describes the cluster level as a function of time occupation $\mathrm{n}_{0}(\mathrm{t})$ and the calculation of the survival probability for the charged cluster characterize the neutralization dynamics. The results are in good agreement with the experimental ones.

\section{Mathematical Model}

Consider the level occupation can be written as:

$$
n_{i j}(t)=\left\langle\psi(t)\left|a_{i}^{+} a_{j}\right| \psi(t)\right\rangle
$$


The wave function $\Psi(\mathrm{t})$ evolve from the initial wave function $\psi\left(t_{o}\right)$ as:

$$
\psi(t)=s\left(t, t_{0}\right) \psi\left(t_{0}\right)
$$

Where,

$$
\frac{\partial s}{\partial t}=-\frac{i}{\hbar} H(t) s\left(t, t_{0}\right)
$$

The Hamiltonian $H(t)$ change with time according to:

$$
H(t)=\sum_{n} \varepsilon_{n}(t) a_{n}^{+} a_{n}+\frac{1}{2} \sum_{n, m}\left\{V_{n m}(t) a_{n}^{+} a_{m}+V_{m n}(t) a_{m}^{+} a_{n}\right\}
$$

We assume that $V_{n n}=0$ then we have $s^{+}\left(t, t_{0}\right)=s^{-1}\left(t, t_{0}\right)$ and

$$
\begin{aligned}
n_{i j}(t) & =\left\langle s\left(t, t_{0}\right) \psi\left(t_{0}\right)\left|a_{i}^{+} a_{j}\right| s\left(t, t_{0}\right) \psi\left(t_{0}\right)\right\rangle \\
& =\left\langle\psi\left(t_{0}\right)\left|\tilde{a}_{i}^{+}(t) \tilde{a}_{j}(t)\right| \psi\left(t_{0}\right)\right\rangle
\end{aligned}
$$

Where the creation operator is defined as

$$
\tilde{a}_{i}^{+}(t) \equiv s^{-1}\left(t, t_{0}\right) a_{i}^{+} s\left(t, t_{0}\right) \text { and } \tilde{a}_{j}^{+}(t) \equiv s^{-1}\left(t, t_{0}\right) a_{j} s\left(t, t_{0}\right)
$$

Then,

$$
\frac{\partial n_{i j}(t)}{\partial t}=\left\langle\psi\left(t_{0}\right)\left|\frac{\partial \tilde{a}_{i}^{+}}{\partial t} \tilde{a}_{j}(t)\right| \psi\left(t_{0}\right)\right\rangle+\left\langle\psi\left(t_{0}\right)\left|\tilde{a}_{i}^{+}(t) \frac{\partial \tilde{a}_{j}}{\partial t}\right| \psi\left(t_{0}\right)\right\rangle
$$

And since

$$
\begin{aligned}
& \frac{\partial \tilde{a}_{n}}{\partial t}=-\frac{i}{\hbar} \varepsilon_{n}(t) \tilde{a}_{n}(t)-\frac{i}{\hbar} \sum_{p} V_{n p}(t) \tilde{a}_{p}(t) \\
& \frac{\partial \tilde{a}_{n}^{+}}{\partial t}=\frac{i}{\hbar} \varepsilon_{n}(t) \tilde{a}_{n}^{+}(t)+\frac{i}{\hbar} \sum_{p} V_{p n}(t) \tilde{a}_{p}^{+}(t)
\end{aligned}
$$

We have,

$$
\begin{array}{r}
\frac{\partial n_{i j}(t)}{\partial t}=\frac{i}{\hbar} \varepsilon_{i}(t) n_{i j}(t)+\frac{i}{\hbar} \sum_{p} V_{p i}(t) n_{p j}(t)-\frac{i}{\hbar} \varepsilon_{j}(t) n_{i j}(t)-\frac{i}{\hbar} \sum_{p} V_{j p}(t) n_{i p}(t) \\
i, j=0,1,2,3 \ldots \mathrm{N} \quad \ldots \ldots \ldots \ldots \ldots \ldots \ldots \ldots \ldots \ldots \ldots \ldots \ldots \ldots \ldots \ldots \ldots \ldots
\end{array}
$$

Or,

$$
i \hbar \frac{\partial n_{i j}(t)}{\partial t}=\left[\varepsilon_{j}(t)-\varepsilon_{i}(t)\right] n_{i j}(t)-\sum_{p}\left\{V_{p i}(t) n_{p j}(t)-V_{j p}(t) n_{i p}(t)\right\}
$$

So we get the set of simultaneous differential equations: 


$$
\begin{aligned}
& \dot{n}_{a a}(t)=-\frac{i}{\hbar} \sum_{k}\left\{V_{a k}(t) n_{a k}(t)-V_{k a}(t) n_{k a}(t)\right\} \\
& \dot{n}_{k k}(t)=\frac{i}{\hbar}\left\{V_{a k}(t) n_{a k}(t)-V_{k a}(t) n_{k a}(t)\right\} \\
& \dot{n}_{a k}(t)=-\frac{i}{\hbar}\left[\varepsilon_{k}-E_{a}(t)\right] n_{a k}(t)-\frac{i}{\hbar} V_{k a}(t) n_{a a}(t)+\frac{i}{\hbar} \sum_{k^{\prime}} V_{k^{\prime} a}(t) n_{k^{\prime} k}(t) \\
& \dot{n}_{k a}(t)=\frac{i}{\hbar}\left[\varepsilon_{k}-E_{a}(t)\right] n_{k a}(t)+\frac{i}{\hbar} V_{a k}(t) n_{a a}(t)-\frac{i}{\hbar} \sum_{k^{\prime}} V_{a k^{\prime}}(t) n_{k k^{\prime}}(t) \\
& \dot{n}_{k^{\prime} k}(t)=-\frac{i}{\hbar}\left[\varepsilon_{k}-\varepsilon_{k^{\prime}}(t)\right] n_{k^{\prime} k}(t)+\frac{i}{\hbar} V_{a k^{\prime}}(t) n_{a k}(t)-\frac{i}{\hbar} V_{k a}(t) n_{k^{\prime} a}(t)
\end{aligned}
$$

We assume that ,

$$
V_{a k}(t)=V_{k a}(t)=V_{k}(t)
$$

the summation of $\boldsymbol{V}_{\boldsymbol{k}}(\boldsymbol{t})=\boldsymbol{v}_{\boldsymbol{k}} \boldsymbol{V}(\boldsymbol{t})$ gives,

$$
\sum_{k}\left|V_{k a}(t)\right|^{2}=V^{2}(t) \int d \varepsilon \rho(\varepsilon)
$$

Then the above equations become:

$$
\begin{aligned}
& \dot{n}_{a a}(t)=-\frac{i}{\hbar} V(t) \int d \varepsilon \rho(\varepsilon)\left[n_{a \varepsilon}(t)-n_{s a}(t)\right] \\
& \dot{n}_{\varepsilon \varepsilon}(t)=\frac{i}{\hbar} V(t)\left[n_{a \varepsilon}(t)-n_{\varepsilon a}(t)\right] \\
& \dot{n}_{\varepsilon^{\prime} \varepsilon}(t)=\frac{i}{\hbar}\left(\varepsilon^{\prime}-\varepsilon\right) n_{\varepsilon^{\prime} \varepsilon}(t)+\frac{i}{\hbar} V(t)\left[n_{a \varepsilon}(t)-n_{\varepsilon^{\prime} a}(t)\right] \\
& \dot{n}_{a \varepsilon}(t)=-\frac{i}{\hbar}\left(\varepsilon-E_{a}(t)\right) n_{a \varepsilon}(t)-\frac{i}{\hbar} V(t)\left[n_{a a}(t)-\int d \varepsilon^{\prime} \rho\left(\varepsilon^{\prime}\right) n_{\varepsilon^{\prime} \varepsilon}(t)\right] \\
& \dot{n}_{\varepsilon a}(t)=\frac{i}{\hbar}\left(\varepsilon-E_{a}(t)\right) n_{\varepsilon a}(t)+\frac{i}{\hbar} V(t)\left[n_{a a}(t)-\int d \varepsilon^{\prime} \rho\left(\varepsilon^{\prime}\right) n_{\varepsilon \varepsilon^{\prime}}(t)\right]
\end{aligned}
$$

The above equations solved by using Runge-Kutta numerical method and Simpson's integral method to get the occupation of the levels. We set $t_{o}=-\infty$ and consider collisions of clusters of $\mathrm{N}$ atoms with mass $M_{A}$ at collision energy $E_{k i n}$, so that $\quad \mathrm{v}^{-2}=\mathrm{M}_{\mathrm{A}} \mathrm{N} / 2 \mathrm{E}_{\text {kin }}$ 


\section{Applications and Results}

\section{A: Platinum $\left(P t_{N}^{-}\right)$clusters}

We test the collision between $P t_{N}^{-}$clusters and two different targets they are:

(i) Highly oriented pyrolytic graphite (HOPG) target with density of state equal to $\mathrm{W}_{\mathrm{HOPG}}=0.74 \mathrm{eV}$ for the peak in the empty region.

(ii) Aluminum (Al) target with density of state equal $\mathrm{W}_{\mathrm{Al}}=5 \mathrm{eV}$ for the peak in the empty region.

The number of state that use in the model $m=51$ state and it is sufficient for the simulation where the increasing $m$ to 500 does not effect on the survival probability $\mathrm{P}_{\mathrm{s}}(\mathrm{N})$.

Figure (1) illustrate the neutralization dynamics of $P t_{N}^{-}$clusters colliding with HOPG at collision energy $E_{k i n}=500 \mathrm{eV}$. This figure presents the occupation of the cluster level as a function of distance. Figure (2) is the same figure (1) but for $\mathrm{Al}$ target.

For HOPG case one can see the occupation of the affinity level $n(t)$ exhibited an oscillating behavior change with cluster size, which mean the charge transfer processes occur many times when the cluster size increase, that is because the large cluster move more slowly than small one, then the neutralization of cluster takes longer.

Figure (2) When aluminum is used as the target, one can see no oscillation has occur, this situation was happen because the band density of state is smoothing more than for HOPG target, this mean when the electron of the $P t_{N}^{-}$cluster jumps to the surface and does not return back to the cluster, in contrast to what happens for HOPG as target. 


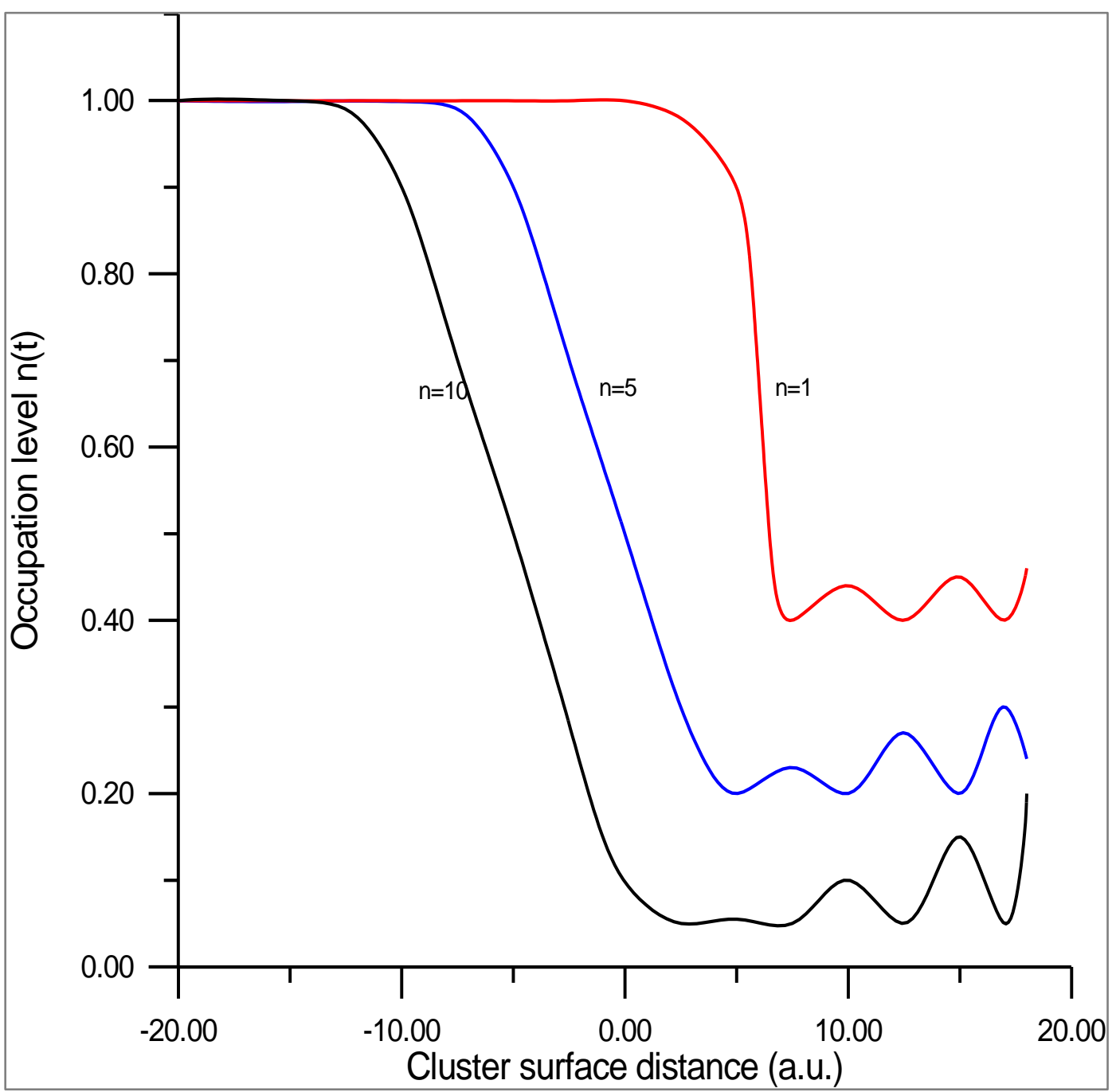

Figure (1): Cluster level occupation $\mathrm{n}(\mathrm{t})$ of $P t_{N}^{-}$cluster during collision with HOPG surface. 


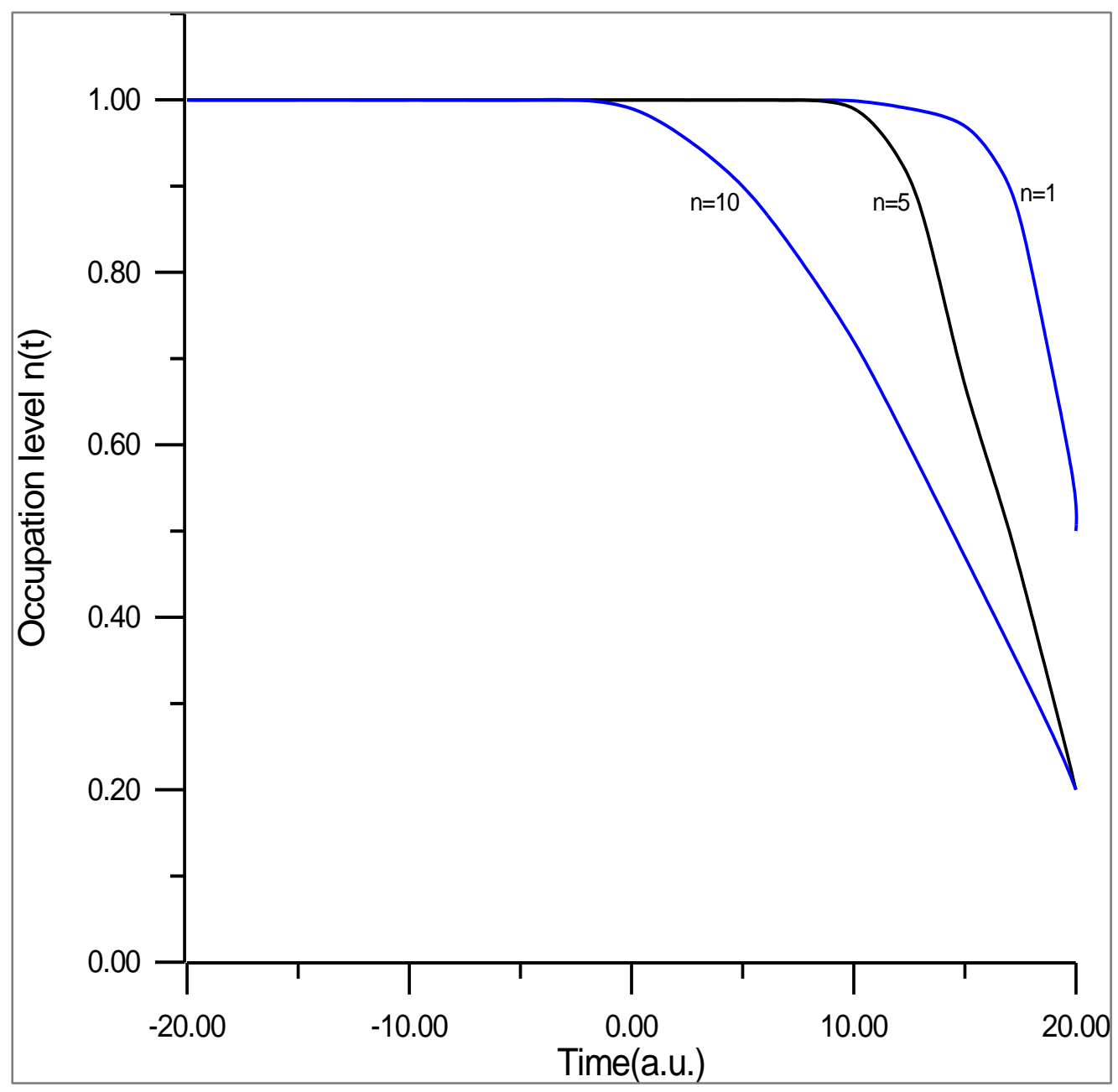

Figure (2): Cluster level occupation $\mathrm{n}(\mathrm{t})$ of $P t_{N}^{-}$cluster during collision with Al surface.

Figure (3) illustrates the survival probability $\mathrm{P}_{\mathrm{S}}(\mathrm{N})$ as a function of cluster size from $\mathrm{N}=1$ to $\mathrm{N}=20$, with incident energy collision of $500 \mathrm{eV}$ when the collision between $P t_{N}^{-}$clusters and HOPG as target: Figure 4 is the same previous figure but the target is the $\mathrm{Al}$, in these figures ( 3 and 4 ) the present works shows a comparison to the theoretical $\mathrm{P}_{\mathrm{s}}(\mathrm{N})$ at time $\mathrm{t}=0$ with the experimentally secondary electron yield $\gamma(N)$ since[ 26],

$\gamma(N) \alpha P_{s}(N, t=0)$ 


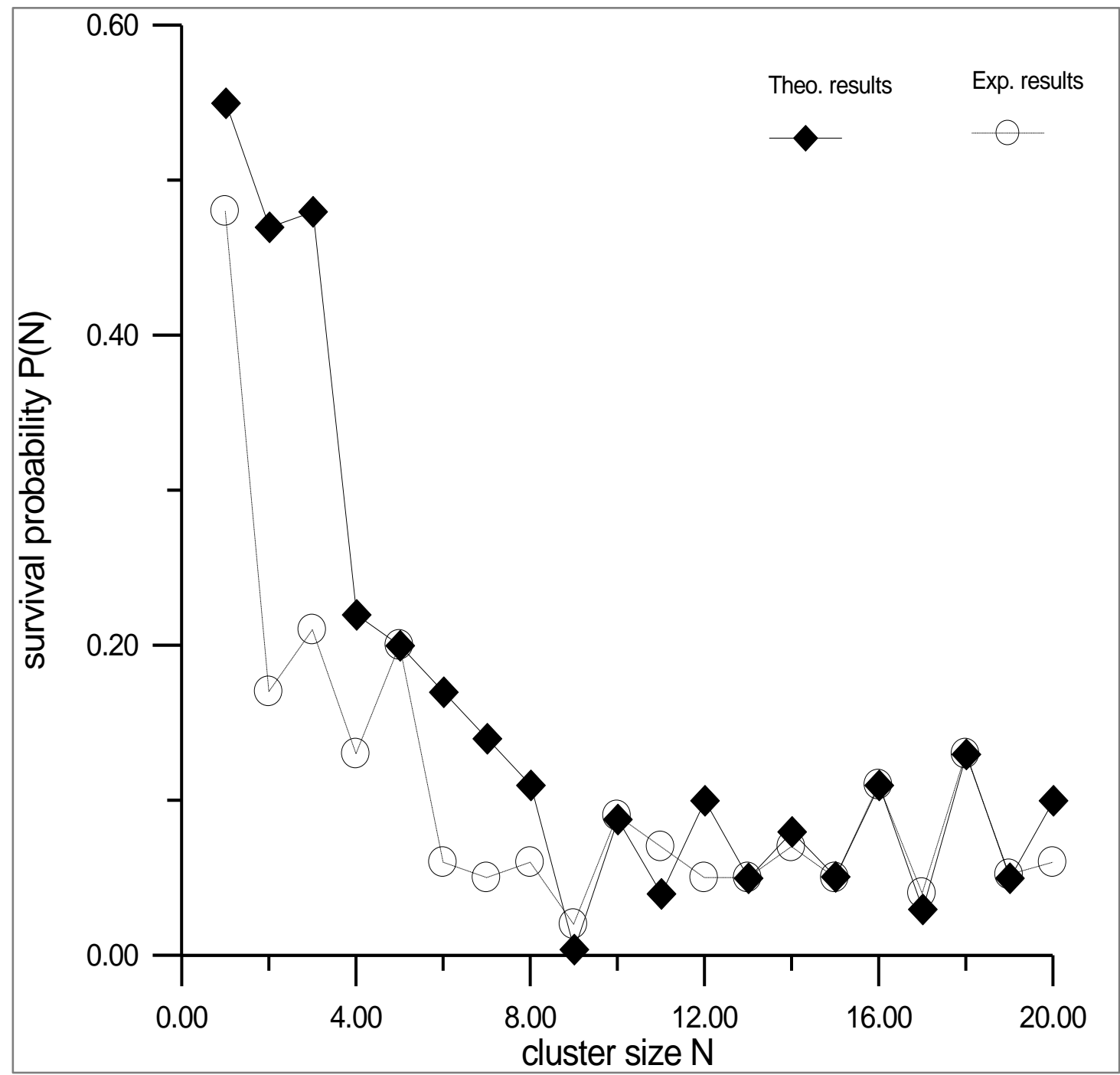

Figure (3): Theoretical and experimental [26] results for the survival probability $\mathrm{P}(\mathrm{N})$ of $P t_{N}^{-}$Clusters impinging on HOPG surface. 


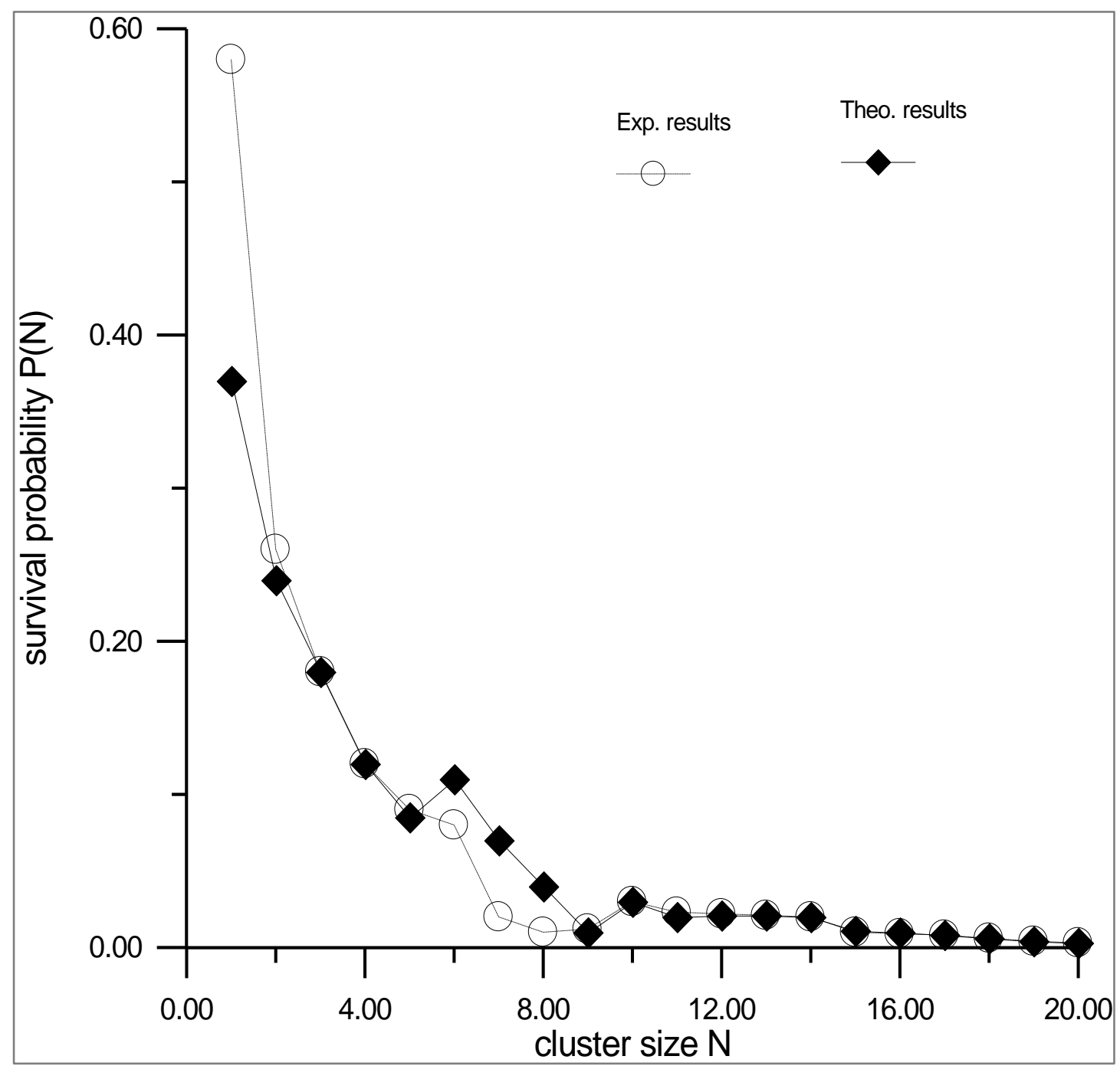

Figure (4): Theoretical and experimental [26] results for the survival probability $\mathrm{P}(\mathrm{N})$ of $P t_{N}^{-}$Clusters impinging on $\mathrm{Al}$ surface.

\section{B: $\mathbf{P b}_{\mathrm{N}}$ clusters}

Figure (5) presents the calculation of the survival probability (theoretical and experimental results) for collisions between $\mathrm{Pb}^{-}$clusters and HOPG surface. One can see the oscillations in the survival probability, This mean that the electrons of the ion jumps to the surface and vise versa. 


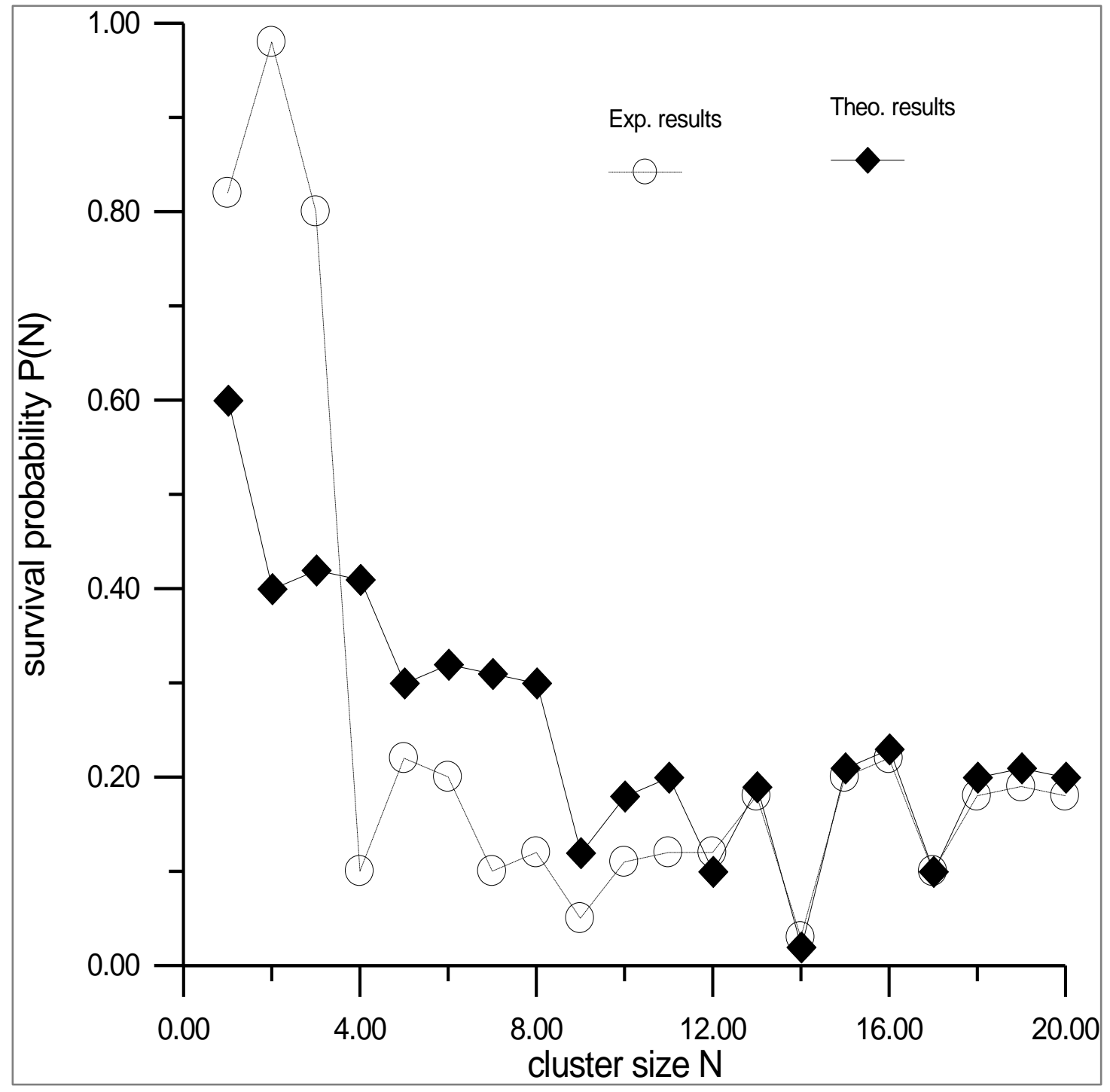

Figure (5): Theoretical and experimental [26] results for the survival probability $\mathrm{P}(\mathrm{N})$ of $\mathrm{Pb}^{-}$Clusters impinging on HOPG surface.

\section{Conclusion}

After we study the survival probability $\mathrm{P}$ as a function of the cluster size $\mathrm{N}$ and time $\mathrm{t}$ (or surface ion distance), when the incident ion is $\mathrm{Pt}_{N}^{-}$and $\mathrm{Pb}^{-}$on the HOPG and $\mathrm{Al}$ surface as a target, one can conclude the following points:

1. The shape of the density of state has the important role on the dynamics of the resonant or quasi resonant charge transfer process i.e. electron of the charging cluster localized or delocalized 
when jumps to the surface, and then HOPG target is different from the Al target since the survival probability has many oscillation.

2. The cluster size is another important parameter, for large $\mathrm{N}$ the charge exchange occur faster than of the small $\mathrm{N}$.

3. The type of the charging cluster play important role in the resonant charge transfer or quasiresonant.

4. The mathematical model that we used is successful and gives us a good agreement with the experimental results. one can learn about the distance dependence of both the affinity level and the interaction potential between cluster and surface.

\section{References}

[1] A. G. Borisov, J. P. Gauyacq and S. V. Shabanov, Surf. Sci., 487, 243 (2001).

[2] H. Winter, C. Auth and A. G. Borisov, Nucl. Instrum. Methods Phys. Res. B, 115, 33 (1996).

[3] C. Auth, A. Mertens, H. Winter, A. G. Borisov and V. Sidis, Phys. Rev. A, 57, 351 (1998).

[4] S. Ustaze, R. Verucchi, S. Lacombe, L. Guillemot and V. A. Esaulov, Phys. Rev. Lett., 79, 3526 (1997).

[5] S. Ustaze, L. Guillemot, R. Verucchi, S. Lacombe and V. A. Esaulov, Nucl. Instrum. Methods. Phys. Res.B, 135, 319 (1998).

[6] A. Mertens, C. Auth, H. Winter and A. G. Borisov, Phys. Rev. A, 55, 846 (1997).

[7] H.H. Brongersma and T.M. Buck, Surf. Sci., 53, 649 (1975).

[8] K.L. Erickson and D.P. Smith, Phys. Rev. Lett., 34, 297 (1975).

[9] A. Blandin, A. Nourtier and D. W. Hone, J. Phys., 37, 369 (1976).

[10] J. C. Tully, Phys. Rev., 16, 4324 (1979).

[11] W. Bloss and D. Hone, Surf. Sci., 72, 277 (1978).

[12] G. Blaise and A. Nourtier, Surf. Sci., 90, 495 (1979).

[13] E. G. Overbosch, R. Rasser, A. D. Tenner and J. Los, Surf. Sci., 92, 310 (1980).

[14] R. Brako and D. M. Newns, Surf. Sci., 108, 253 (1981).

[15] K.L. Sabastian, V. C. Jyothi Bhasu and T. B. Grimley, Surf. Sci., 110,1571(1981).

[16] R. Brako and D. M. Newns, Rep. Prog. Phys., 52, 655 (1989).

[17] A. G. Borisov, V. Sidis and H. Winter, Phys. Rev. Lett., 77, 1893 (1996).

[18] L. Guillemot and V. A. Esaulov, Phys. Rev. Lett. 82, 4552 (1999).

[19] S. B. Hill, C. B. Haich, Z. Zhou, P. Nordlander, and F. B. Dunning, Phys. Rev. Lett., 85, 5444 (2000).

[20] S. Ustaze et al., Surf. Sci. 415, 1027 (1998).

[21] A. Robin, D. Niemann, N. Stolterfoht, and W. Heiland, Phys.Rev. A 67, 052901 (2003).

[22] A.A. Saleh, V. Shutthanandan, N.R. Shivaparan, R.J. Smith,T.T. Tran and S.A. Chambers, Phys. Rev. B, 56, 9841 (1997).

[23] A. Murgaza, A. Mascaraque, V. Pérez-Dieste, V. Repain, S.Rousset, F. J. García de Abajo, and J. E. Ortega, Phys. Rev.Lett. 87, 107601 (2001).

[24] F. Schiller, M. Ruiz-Osés, J. Cordón, and J. E. Ortega, Phys. Rev. Lett. 95, 066805 (2005).

[25] I.F. Urazgil'din and A.G. Borisov, Vacuum, 40, 461 (1990).

[26] O. Speer, M.E. Garcia, Bu. Wrenger and K.H. Meiwes-Broer, surf. Sci.,443, 195 (1999). 